Article

\title{
Taqman qPCR Quantification and Fusarium Community Analysis to Evaluate Toxigenic Fungi in Cereals
}

\author{
Elina Sohlberg ${ }^{1, * \mathbb{C}}$, Vertti Virkajärvi ${ }^{1}$, Päivi Parikka ${ }^{2}$, Sari Rämö ${ }^{2}$, Arja Laitila ${ }^{1}$ and Tuija Sarlin ${ }^{1}$ \\ 1 VTT Technical Research Centre of Finland Ltd., FI-02044 Espoo, Finland; vertti@no-office.co (V.V.); \\ Arja.Laitila@outlook.com (A.L.); tuija.sarlin@vtt.fi (T.S.) \\ 2 Natural Resources Institute Finland (Luke), FI-31600 Jokioinen, Finland; ext.paivi.parikka@luke.fi (P.P.); \\ sari.ramo@luke.fi (S.R.) \\ * Correspondence: elina.sohlberg@vtt.fi
}

check for updates

Citation: Sohlberg, E.; Virkajärvi, V.; Parikka, P.; Rämö, S.; Laitila, A.;

Sarlin, T. Taqman qPCR

Quantification and Fusarium

Community Analysis to Evaluate Toxigenic Fungi in Cereals. Toxins 2022, 14, 45. https://doi.org/ $10.3390 /$ toxins 14010045

Received: 9 December 2021

Accepted: 4 January 2022

Published: 6 January 2022

Publisher's Note: MDPI stays neutral with regard to jurisdictional claims in published maps and institutional affiliations.

Copyright: (C) 2022 by the authors. Licensee MDPI, Basel, Switzerland. This article is an open access article distributed under the terms and conditions of the Creative Commons Attribution (CC BY) license (https:// creativecommons.org/licenses/by/ $4.0 /)$.
Abstract: Fusarium head blight (FHB) is an economically important plant disease. Some Fusarium species produce mycotoxins that cause food safety concerns for both humans and animals. One especially important mycotoxin-producing fungus causing FHB is Fusarium graminearum. However Fusarium species form a disease complex where different Fusarium species co-occur in the infected cereals. Effective management strategies for FHB are needed. Development of the management tools requires information about the diversity and abundance of the whole Fusarium community. Molecular quantification assays for detecting individual Fusarium species and subgroups exist, but a method for the detection and quantification of the whole Fusarium group is still lacking. In this study, a new TaqMan-based qPCR method (FusE) targeting the Fusarium-specific elongation factor region (EF1 $\alpha)$ was developed for the detection and quantification of Fusarium spp. The FusE method was proven as a sensitive method with a detection limit of 1 pg of Fusarium DNA. Fusarium abundance results from oat samples correlated significantly with deoxynivalenol (DON) toxin content. In addition, the whole Fusarium community in Finnish oat samples was characterized with a new metabarcoding method. A shift from F. culmorum to F. graminearum in FHB-infected oats has been detected in Europe, and the results of this study confirm that. These new molecular methods can be applied in the assessment of the Fusarium community and mycotoxin risk in cereals. Knowledge gained from the Fusarium community analyses can be applied in developing and selecting effective management strategies for FHB.

Keywords: Fusarium; mycotoxins; DON; qPCR; NGS; Fusarium head blight; cereals

Key Contribution: This study developed a new qPCR method and applied a novel metabarcoding method for the detection and quantification of Fusarium spp. Fusarium abundance results measured with the FusE qPCR method correlated significantly with deoxynivalenol (DON) toxin content.

\section{Introduction}

Fusarium head blight (FHB), a devastating plant disease that infects wheat, barley, oats, and other small grain cereals, causes billions of dollars of crop yield and quality losses worldwide [1-3]. Up to 17 fungal species have been shown to cause FHB, among which most species belong to the genus Fusarium, and a couple of species belong to genus $\mathrm{Mi}$ crodochium [4,5]. Often, Fusarium species form a disease complex where different Fusarium species co-occur in the infected cereals [6]. Some Fusarium species produce mycotoxins that cause a risk for the end-use of the cereal crops in the food and feed industry. One especially important plant pathogen is Fusarium graminearum, which produces the mycotoxins trichothecenes deoxynivalenol (DON) and nivalenol (NIV) and the mycoestrogen zearalenone (ZEN) [7]. The identification of species that inflict FHB is important, since they differ in fungicide sensitivity, host resistance, pathogenicity, and toxin production [8]. In Finnish 
cereals, the most common Fusarium species detected in 2005-2014 were F. avenaceum, F. culmorum, F. graminearum, F. poae, F. sporotrichioides, and F. langsethiae [9]. The prevalence of F. culmorum and F. graminearum was high in oats and barley, but when compared to previous studies, a shift from F. culmorum to F. graminearum was detected, which has led to increased DON content. A rapid increase in the prevalence of F. graminearum has also been detected in other European countries [10-12]. Effective FHB management strategies are needed and the development of these management methods requires information about the composition and abundance of the whole Fusarium community, because different populations could be affected selectively [13]. To evaluate the impact of fungal control means in disease control, quantitative data of the Fusarium community and different species are needed.

A variety of different methods are currently applied in the detection and identification of Fusarium species in cereals-for a recent review, see Karelov et al. [14]. Traditional plating methods on a selective nutrient medium applied in the detection of Fusarium fungi are laborious and some species can outgrow others, preventing the detection of the whole Fusarium community. In the past few years, the focus in Fusarium detection has been on molecular DNA-based methods. The most prevalent DNA-based methods for Fusarium quantification and identification are single and multiplex qPCR methods that detect different Fusarium-specific DNA regions. Bluhm et al. [15] developed a TaqMan realtime PCR assay for group-specific detection of trichothecene- and fumonisin-producing Fusarium spp. by targeting the genes involved in mycotoxin biosynthesis, and Sarlin et al. [16] evaluated the application of the method of Fusarium trichothecene gene-region measurement to detect toxigenic fungi in barley and malt. In addition, Kulik et al. [17] developed a TaqMan-based qPCR assay targeting the enniatin synthetase gene to quantify Fusarium avenaceum, Fusarium tricinctum, and Fusarium poae abundance in wheat grains, and a positive and significant correlation was observed between the Fusarium amount and the total concentration of enniatins. Waalwijk et al. [8] reported the development and application of a TaqMan qPCR method to quantify individual Fusarium species and closely related Microdochium nivale in infected cereals. Recently, Morcia et al. [5] applied a new chip digital PCR method for quantification of F. graminearum, F. culmorum, F. sporotrichioides, F. poae, and F. avenaceum. Then, Sonia et al. [17] developed individual qPCR methods for seven Fusarium and two Microdochium species. The co-occurrence of several Fusarium species in cereals makes monitoring more challenging, and the detection of only a few Fusarium species is not sufficient. Genus-specific conventional PCR methods are available for the detection of the presence of Fusarium fungi in cereals [14], but a genus-specific quantitative PCR method for quantification of all the Fusarium species is still lacking.

New metabarcoding methods using the Fusarium elongation factor region (EF1 $\alpha$ gene) have been applied in assessing the diversity of the Fusarium community in cereals [18,19]. These new metabarcoding methods enable the detection of the Fusarium community to the species level and monitoring the distribution and community shifts in cereals in changing environmental conditions. In addition, new emerging species can be detected with these methods.

In this study, a new TaqMan-based qPCR method (FusE) targeting the Fusariumspecific elongation factor region $(E F 1 \alpha)$ was developed for the detection and quantification of whole Fusarium spp. simultaneously. As a proof of principle, new FusE qPCR analyses were performed on field samples to assess the Fusarium contamination in Finnish oat crop survey samples collected in 2015-2017. The years 2016 and 2017 were especially challenging regarding the Fusarium contamination, and 25\% of the national yearly crop survey oat samples collected by the Finnish Cereal Committee had mycotoxin deoxynivalenol (DON) content over $1750 \mu \mathrm{g} / \mathrm{kg}$. To evaluate the connection between Fusarium abundance and DON content, the correlation between the FusE qPCR, Fusarium plating method, and deoxynivalenol (DON) results was evaluated. In addition, the whole Fusarium community in oat samples was characterized with a novel Fusarium metabarcoding method. 


\section{Results}

\subsection{FusE qPCR Method}

The amplification with the newly designed FusE primers and probe confirmed a highly specific amplification, and only one single product with a size of $96 \mathrm{bp}$ was amplified with the FusE primers. All 14 Fusarium species tested (Table 1) were amplified with the primer set. Other tested fungi, yeast, and bacteria associated and isolated from cereal samples (Table 1) were not detected with the primes. In addition to Fusarium species, Microdochium nivale, and M. majus strains were amplified with FusE primers.

Table 1. Fungal and bacterial isolates and their origins.

\begin{tabular}{|c|c|c|c|}
\hline Microbial Group & VTT-Strain & Species & Origin/Host, Country \\
\hline \multirow{16}{*}{ Fusarium-species: } & VTT D-80141 & F. avenaceum & $\begin{array}{l}\text { Barley, } \\
\text { Finland }\end{array}$ \\
\hline & VTT D 96601 & $\Gamma$ corealis & Barley, \\
\hline & 211 D-96601 & F. cerealts & Finland \\
\hline & VTT D-80148 & E culmorum & Barley, \\
\hline & & & Finland \\
\hline & VTT D-82087 & F. equiseti & $\begin{array}{c}\text { Rotting fruit of } \\
\text { Cucumis melo, Turkey }\end{array}$ \\
\hline & VTT D-82082 & F. graminearum & $\begin{array}{c}\text { Barley, } \\
\text { Finland }\end{array}$ \\
\hline & VTT D-95470 & F. graminearum & Corn, USA \\
\hline & VTT D-03931 & F. langsethiae & Barley, \\
\hline & VTT D-80134 & F. oxysporum & Grain \\
\hline & VTT D-76038 & F. poae & Barley \\
\hline & VTT D-82182 & F. poae & $\begin{array}{l}\text { Barley, } \\
\text { Germany }\end{array}$ \\
\hline & VTT D-77056 & F. sambucinum & Grain \\
\hline & VTT D-77057 & F. solani & Grain \\
\hline & VTT D-72014 & F. sporotrichioides & Grain \\
\hline & VTT D-131559 & F. tricinctum & $\begin{array}{l}\text { Barley, } \\
\text { Finland }\end{array}$ \\
\hline \multirow{13}{*}{ Other fungi: } & VTT D-96653 & Achremonium polychronum & Mouldy house, Finland \\
\hline & VTT D-071272 & Aureobasidium pullulans & Native wheat bran, Finland \\
\hline & VTT D-76024 & Alternaria alternata & Barley \\
\hline & VTT D-00808 & Aspergillus ochraceus & $\begin{array}{l}\text { Barley, } \\
\text { Finland }\end{array}$ \\
\hline & VTT D-97673 & $\begin{array}{c}\text { Clonostachys rosea } f . \\
\text { catenulata }\end{array}$ & Soil, Finland \\
\hline & VTT D-76039 & Cochliobolus sativus & $\begin{array}{l}\text { Barley, } \\
\text { Finland }\end{array}$ \\
\hline & VTT D-03923 & Eurotium amstelodami & Barley, \\
\hline & & Eurottum amstetoaаm & Finland \\
\hline & VTT D-94425 & Geotrichum candidum & Malting process, Finland \\
\hline & VTT D-131555 & Microdochium nivale & Feed barley, Finland \\
\hline & VTT D-131551 & Microdochium nivale & Wheatfield soil, the Netherlands \\
\hline & VTT D-131552 & Microdochium majus & Winter wheat, USA \\
\hline & VTT D-99750 & Penicillium verrucosum & Barley, Denmark \\
\hline Yeasts: & VTT C-92011 & Rhodotorula glutinis & Barley malting, Finland \\
\hline \multirow{2}{*}{ Bacteria: } & VTT E-93497 & Leuconostoc citreum & Malting process, Finland \\
\hline & VTT E-90398 & Pantoea agglomerans & Barley, Finland \\
\hline
\end{tabular}

Linearity, efficiency, and the theoretical limit of detection were estimated from the standard curve with Fusarium genomic DNA ranging from 1 to $0.001 \mathrm{ng}$ (Figure 1). The cycle threshold $(\mathrm{Ct})$ values from the standard curve showed a linear dynamic range, corresponding to a $\mathrm{Ct}$ range of 23 to 34 cycles. The lower detection limit of the Fusarium 
spp. was $0.001 \mathrm{ng}$ of Fusarium genomic DNA per reaction, as 35 cycles was set to be the cutoff value due to uncertainty of the last five cycles. Linear regression between the amount of Fusarium DNA (ng) and the corresponding Ct values revealed $\mathrm{r}^{2}$ values of 1 , while the mean efficiency of the qPCR was 95\% $(n=10)$. No PCR inhibition was observed when a known amount of Fusarium genomic DNA was added to the DNA sample containing the cereal matrix (data not shown).

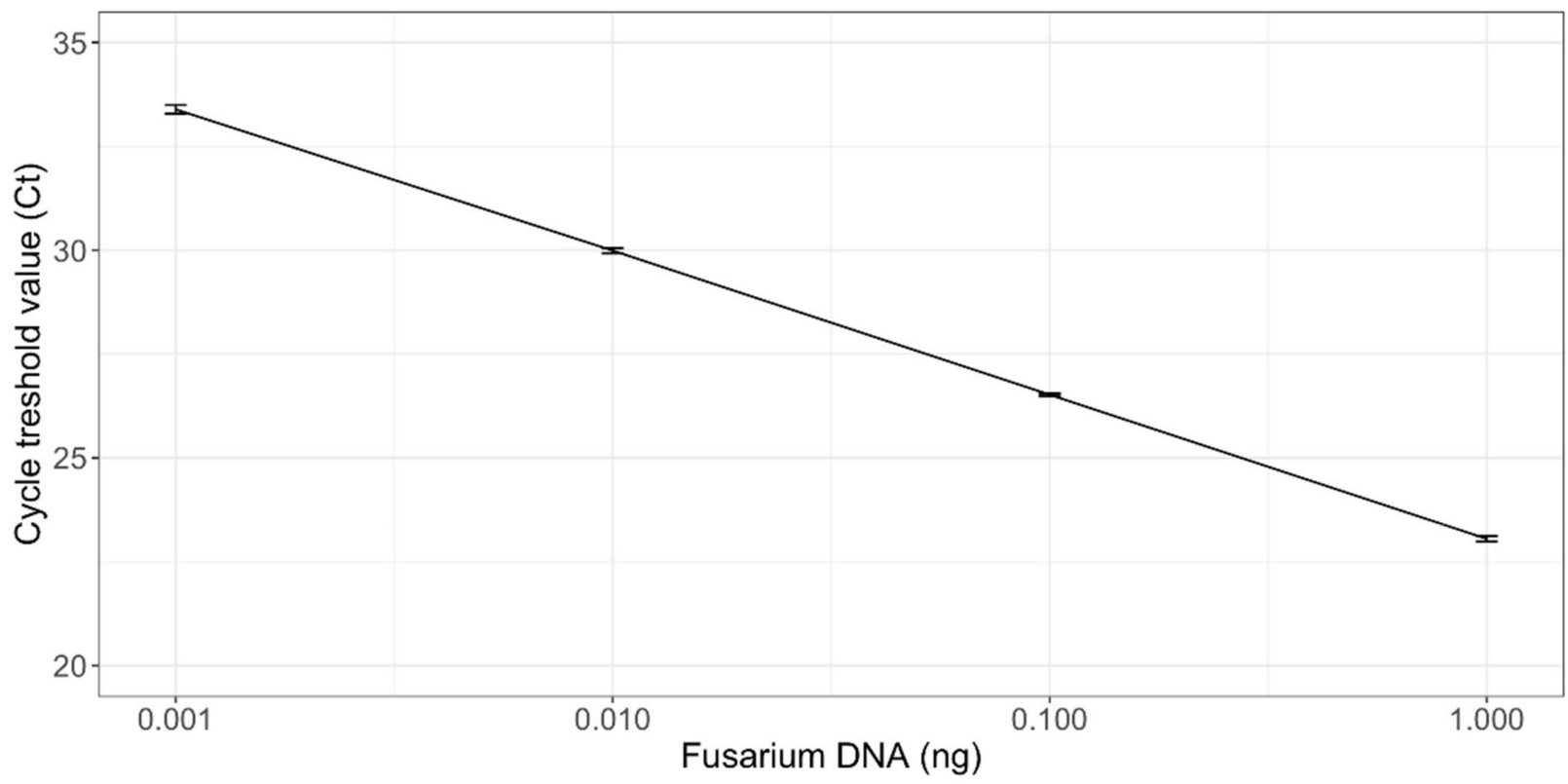

Figure 1. Standard curve of the FusE qPCR assay showing Fusarium DNA concentration (ng) against the cycle threshold $(\mathrm{Ct})$ values of a single $\mathrm{qPCR}$ run. The Fusarium DNA concentration range was 0.001 to $1 \mathrm{ng}$ per reaction. The amount of Fusarium DNA $(0.001-1 \mathrm{ng})$ on the $X$-axis was plotted against the $\mathrm{Ct}$ values from 20 to 35 on the $Y$-axis. Linear regression equation of the standard curve was $Y=-3.45 x+23.05$ at $r^{2}=1$. The efficiency was $95 \%$. The standard deviation of three technical replicates is presented on vertical bars.

\subsection{Deoxynivalenol Levels in Oat Samples}

The deoxynivalenol (DON) content in oat grain varied substantially between the samples collected in different years (Figure 2). In 2015, the DON content ranged from 0 to $2700 \mu \mathrm{g} / \mathrm{kg}$, and the average $(n=9)$ was $1103 \mu \mathrm{g} / \mathrm{kg}$. In 2016 and 2017, the higher DON contamination of grain was detected, and the content of this mycotoxin varied significantly between the samples. In 2016, the DON content ranged from 130 to 26,000 $\mu \mathrm{g} / \mathrm{kg}$ and the average $(n=27)$ was $5334 \mu \mathrm{g} / \mathrm{kg}$, whereas in 2017, the DON content varied between 310 and $23,000 \mu \mathrm{g} / \mathrm{kg}$ and the average $(n=15)$ was $6620127 \mu \mathrm{g} / \mathrm{kg}$. 


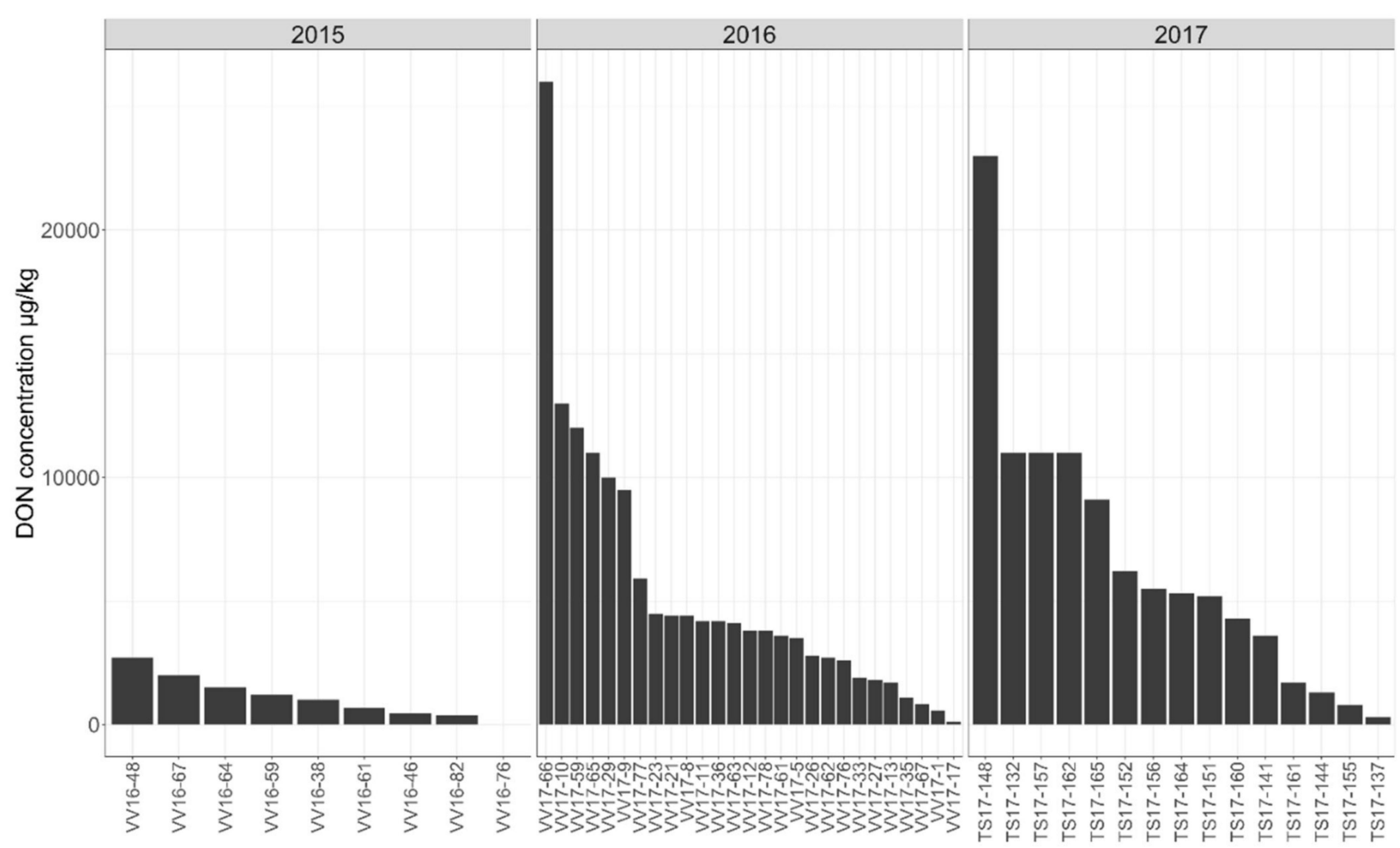

Figure 2. The content of mycotoxin deoxynivalenol (DON) in the oat samples in years 2015-2017.

\subsection{Fusarium Abundance in Field Samples by a Conventional Plating Method}

Various Fusarium species were detected from oat samples in the years 2015-2017 by the plating method (Figure 3). The most abundant fungi were F. graminearum and F. avenaceum. In the year 2015, the percentage of F. graminearum- and F. avenaceum-contaminated grains in oat samples varied from $20 \%$ to $56 \%$, with an average $(n=9)$ of $35 \%$, and from $2 \%$ to $31 \%$, with an average of $12 \%$, respectively. Other minor Fusarium species detected in 2015 were F. arthrosporioides, F. culmorum, F. tricinctum, F. poae, F. sporotrichioides, F. langsethiae, and F. sambucinum. In 2016, the F. graminearum contamination rate varied between $2 \%$ and $87 \%$, with an average $(n=27)$ of $51 \%$, and the F. avenaceum contamination rate varied between $3 \%$ and $55 \%$, with an average of $25 \%$. Other minor Fusarium species cultivated were $F$. arthrosporioides, F. culmorum, F. tricinctum, F. poae, F. sporotrichioides, F. langsethiae, F. equiseti, and F. sambucinum. In 2017, the percentage of grains contaminated with F. gramiearum plating method varied between $27 \%$ and $80 \%$, with an average $(n=15)$ of $61 \%$, and the percentage of those contaminated with F. aveneceum varied between $0 \%$ and $28 \%$, with an average of $8 \%$. Other minor Fusarium species detected by the plating method were $F$. arthrosporioides, F. culmorum, F. tricinctum, F. poae, F. sporotrichioides, F. langsethiae, and F. sambucinum. 


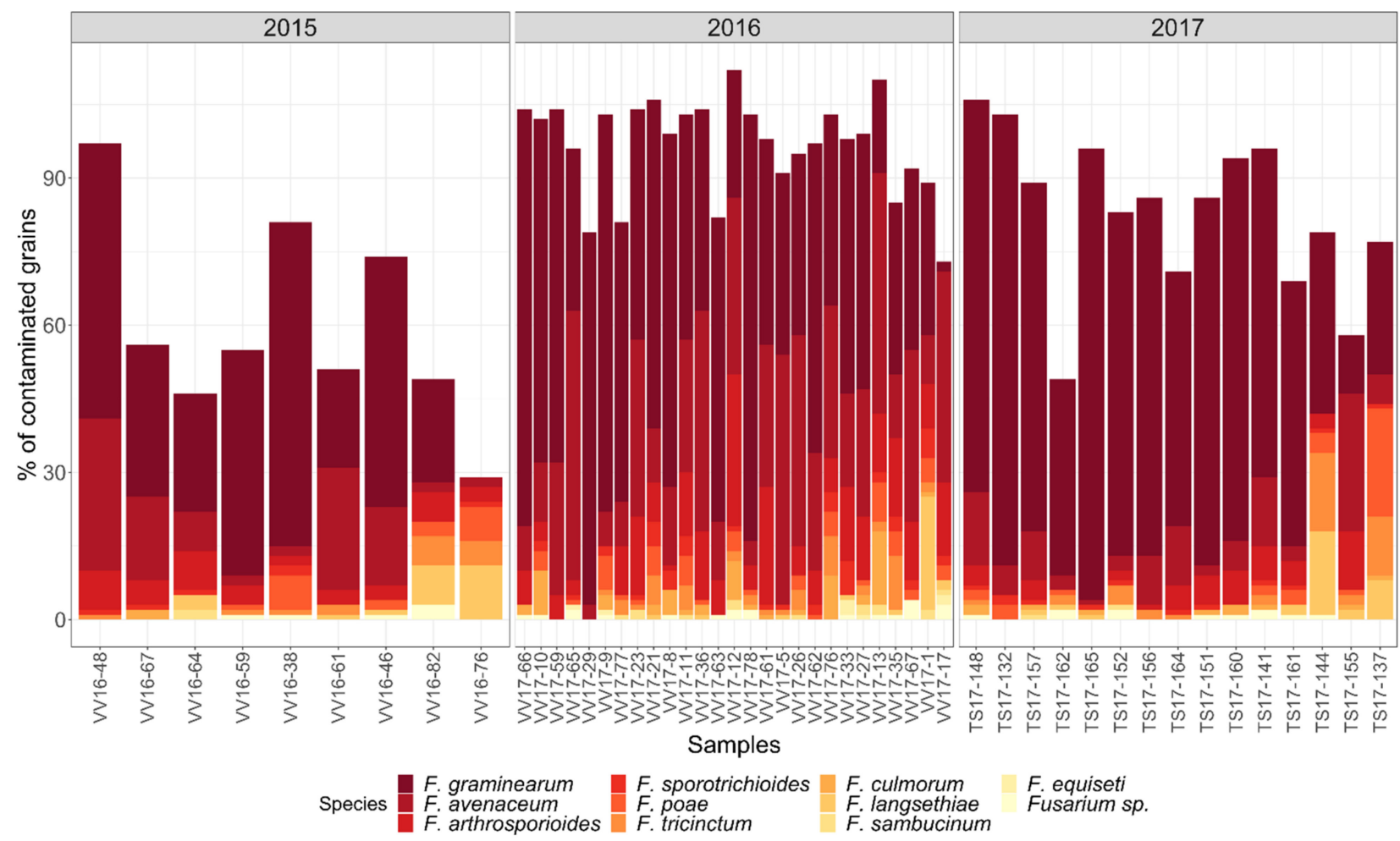

Figure 3. Fusarium species abundance in oat samples from the years 2015-2017 was detected with the plating method. Fusarium species abundance is shown as the percentage (\%) of contaminated grains.

\subsection{Fusarium Abundance Detected with FusE qPCR}

Low Fusarium DNA levels in field samples ranging from 0.3 to $2.4 \mathrm{pg}$ of Fusarium DNA $\mathrm{ng}^{-1}$ of total DNA with average $(n=9)$ of $1.1 \pm 0.6 \mathrm{pg}$ of Fusarium DNA $\mathrm{ng}^{-1}$ of total DNA were detected in 2015 (Figure 4). F. graminearum and F. culmorum levels were not measured from the 2015 field samples, because of low Fusarium spp. contamination levels. In 2016 and 2017, cereal cultivation conditions promoted Fusarium growth, and increased Fusarium abundance was measured. The Fusarium DNA levels in oat samples in 2016 and 2017 were triple compared to the year 2015. In 2016, the Fusarium spp. DNA levels ranged from 1.3 to $16.1 \mathrm{pg}$ of Fusarium DNA ng ${ }^{-1}$ of total DNA with an average $(n=27)$ of $5.3 \pm 3.7 \mathrm{pg}$ of Fusarium DNA ng ${ }^{-1}$ of total DNA. In 2017, the Fusarium spp. abundance ranged from 3.2 to 15.5 , with an average $(n=15)$ of $8.5 \pm 3.9$ pg of Fusarium DNA ng ${ }^{-1}$ of total DNA. The variation of the Fusarium DNA levels was substantial between the samples in 2016 and 2017. F. graminearum abundance in samples from the year 2016 was high, ranging from 0.4 to 16.3 , with an average $(n=27)$ of $3.6 \pm 3.5 \mathrm{pg}$ of . graminearum DNA ng ${ }^{-1}$ of total DNA. In addition, small amounts of F. culmorum were detected in some of the samples, ranging from 0.0001 to $0.24 \mathrm{pg}$ of F. culmorum DNA $\mathrm{ng}^{-1}$ of total DNA. In the year 2017, high amounts of $F$. graminearum DNA were detected again, ranging from 3.2 to 8.1, with an average $(n=15)$ of $2.6 \pm 2.2 \mathrm{pg}$ of F. graminearum DNA $\mathrm{ng}^{-1}$ of total DNA. No F. culmorum was detected in the samples collected in 2017. 


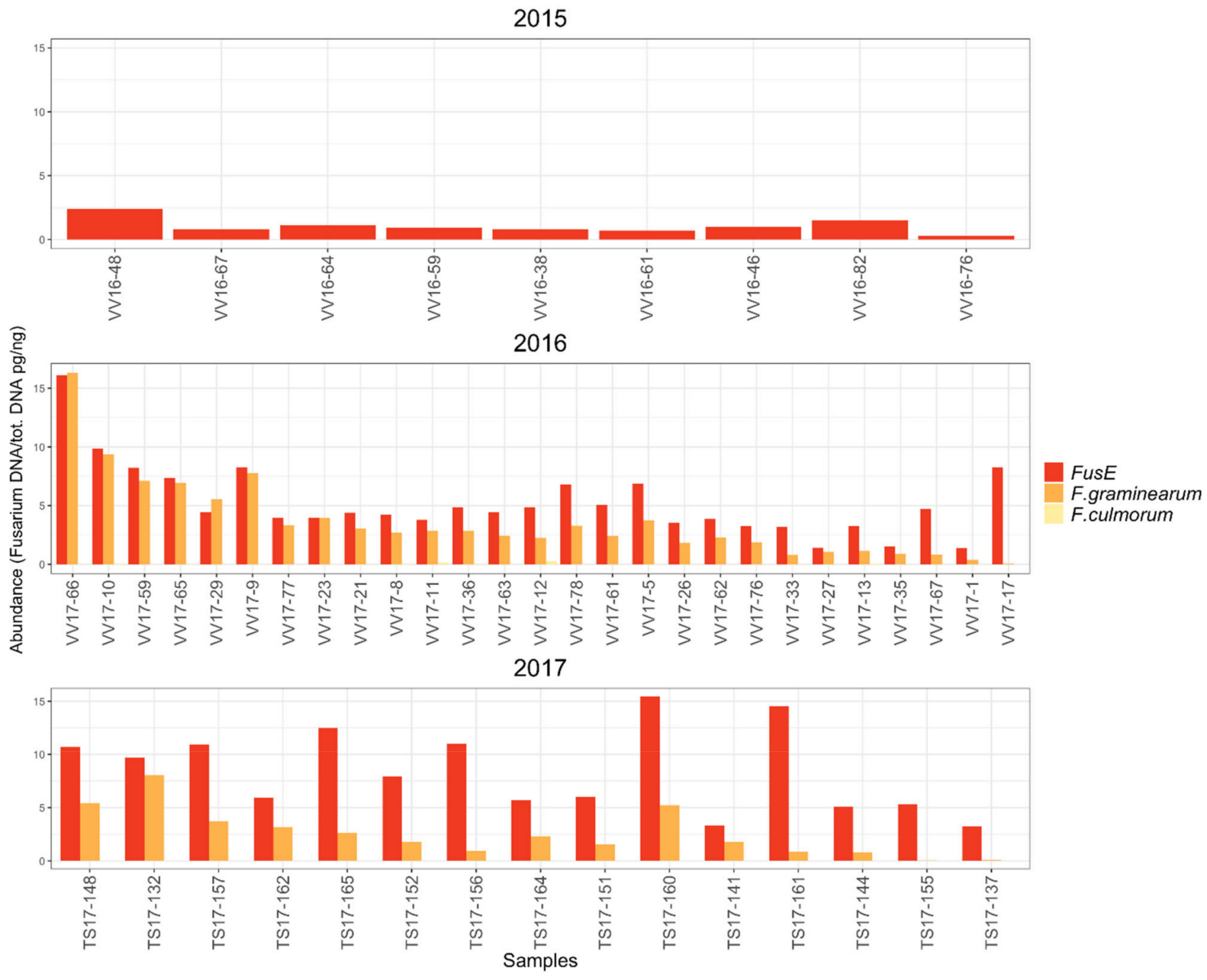

Figure 4. Amount of Fusarium spp. DNA, F. graminearum DNA, and F. culmorum DNA in the oat samples from the years 2015-2017. In 2015, only Fusarium spp. analysis was performed because contamination with F. graminearum and F. culmorum was low according to the Fusarium plating method.

\subsection{Fusarium Community in Field Samples}

The Fusarium community diversity in oat samples was analyzed with next-generation sequencing. A total number of 893,699 sequences of the Fusarium elongation factor region were obtained from Illumina Miseq (Illumina, Inc., San Diego, CA, USA) sequencing after quality control. These sequences were divided into 420 amplicon sequence variants (ASVs), from which 267 ASVs belonged to the genus Fusarium. The dominant species was Microdochium nivale, which is a close relative to Fusarium species and has previously been associated with the Fusarium genus (Supplementary Materials, Figure S1). Species that did not belong to the Fusarium genus were extracted from the data to study the Fusarium community in more detail. The Fusarium community structure varied between the years (Figure 5). Based on the community profiling, the most abundant species in the 2015 oat crop was F. oxysporum, with relative abundance ranging from $9 \%$ to $99 \%$ of the total community in the samples. In one sample (VV16_38), the dominant species was F. avenaceum (76\%). In 2015, F. graminearum was found as a minor group, and relative abundance ranged from 0 to $26 \%$. Other Fusarium species detected in 2015 were F. poae and F. langsethiae. In 2016, the dominant Fusarium species were F. oxysporum (2-87\%) and F. graminearum (0-87\%). Other Fusarium species detected were F. avenaceum, F. langsethiae, F. poae, F. tricinctum, and F. equiseti. In addition, strain NRRL 25,130 detected in sample VV17-13 has been identified as Fusarium avenaceum [20]. In 2017, F. oxysporum was the dominant Fusarium species, and 
the relative abundance ranged from $1.5 \%$ to $82 \%$, but F. graminearum was also detected in the most of samples (0-88\%), and was the dominant species in two oat samples. Other Fusarium species detected from the oat samples were F. poae, F. avenaceum, and F. langsethiae.

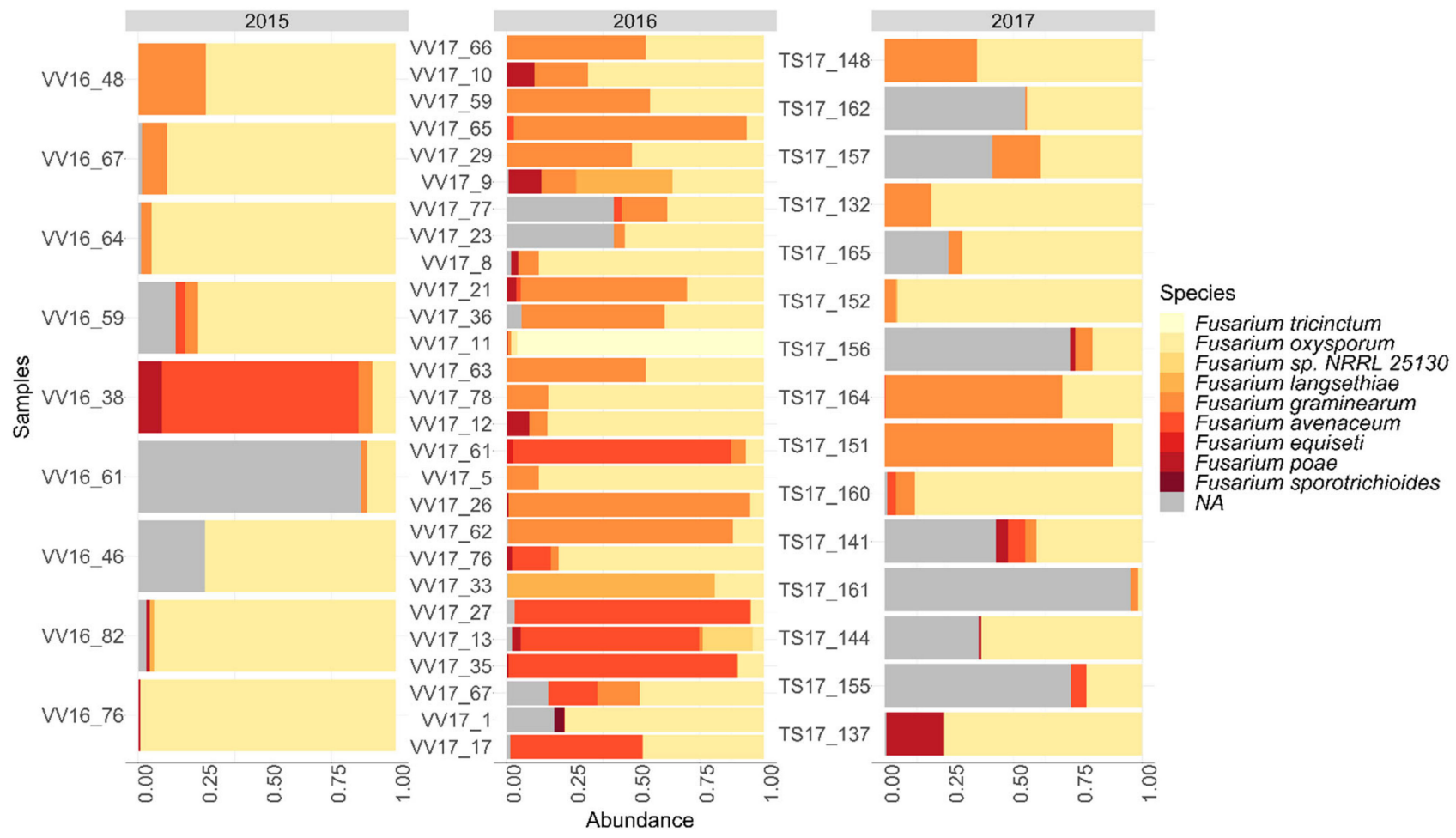

Figure 5. Relative abundance (\%) of Fusarium species detected with the metabarcoding method in the oat samples in the years 2015-2017. NA: Fusarium spp., not identified to the species level.

\subsection{Correlation of Fusarium Abundance and DON Levels}

The correlation of the Fusarium abundance in oat samples detected with the FusE qPCR method and DON content was analyzed. In addition, the correlation of qPCR results and Fusarium abundance detected with the plating method was analyzed. The correlation was calculated from the whole sample set consisting of 157 oat samples and 40 barley samples from which the FusE assay, species-specific qPCR assays, a conventional Fusarium plating method, and DON measurement were performed. Based on the statistical analysis, the DON content correlated significantly ( $p$-value $<0.01)$ with the amount of Fusarium spp. DNA detected with the FusE qPCR assay and with the amount of F. graminearum DNA detected with the F. graminearum specific qPCR assay in all three years studied (Figure 6). Fusarium spp. qPCR results and F. graminearum qPCR results also correlated significantly $(p$-value $<0.01)$ with the F. graminearum plating method results. 


\begin{tabular}{|c|c|c|c|c|c|c|c|}
\hline & $\begin{array}{l}\text { Fusarium } \\
\text { DNA }\end{array}$ & $\begin{array}{c}\text { Fusarium } \\
\text { DNA }\end{array}$ & $\begin{array}{c}\text { F. graminearum } \\
\text { DNA }\end{array}$ & $\begin{array}{c}\text { F.culmorum } \\
\text { DNA }\end{array}$ & $\begin{array}{c}\text { Fusarium } \\
\text { DNA }\end{array}$ & $\begin{array}{c}\text { F. graminearum } \\
\text { DNA }\end{array}$ & $\begin{array}{c}\text { F.culmorum } \\
\text { DNA }\end{array}$ \\
\hline Crop year & 2015 & 2016 & 2016 & 2016 & 2017 & 2017 & 2017 \\
\hline$n$ & 78 & 82 & 82 & 82 & 37 & 37 & 37 \\
\hline DON & +++ & +++ & +++ & & +++ & +++ & \\
\hline \multicolumn{8}{|l|}{ F. avenaceum } \\
\hline F. culmorum & & & & +++ & + & + & \\
\hline F. graminearum & +++ & +++ & +++ & & +++ & +++ & \\
\hline \multicolumn{8}{|l|}{ F. sporotrichioides } \\
\hline F. poae & & & & & - & & \\
\hline F. tricinctum & & - & - & & & - & \\
\hline F. arthrosporioides & + & & - & +++ & & & \\
\hline \multicolumn{8}{|l|}{ F. langsethiae } \\
\hline Total Fusarium species & + & +++ & +++ & & +++ & +++ & \\
\hline
\end{tabular}

Figure 6. Correlation of the different Fusarium qPCR methods, DON content, and plating method of Fusarium species. Fusarium DNA indicates the results of the FusE qPCR assay and the F. graminearum DNA and F. culmorum DNA species-specific qPCR assays results. The correlation was analyzed by the calculation of Pearson or Spearman correlation coefficients at the significance levels of $p<0.05$ and $p<0.01$. The number of + and - signs indicates the levels of significance of the positive and negative correlation, respectively: + or,$- p<0.05$, and,$+++ p<0.01$.

\section{Discussion}

An efficient and rapid method for Fusarium quantification is needed for assessing the FHB and mycotoxin risk in cereals. Quantitative PCR assays with TaqMan-based primers for specific individual Fusarium species and some toxin-producing Fusarium species have been developed $[8,15,17]$. However, an efficient method for quantifying the whole Fusarium group with one qPCR method has been lacking so far. The co-occurrence of several Fusarium species in the FHB complex in cereals makes monitoring challenging. If only a few Fusarium species are targeted with qPCR, the Fusarium contamination can remain underestimated or even undetected.

This study developed a new TaqMan qPCR assay targeting the Fusarium elongation factor region $(E F 1 \alpha)$ to quantify the abundance of Fusarium spp. in cereal samples. To validate the method with representative material, DNA from 157 oat samples and 40 barley samples were extracted. The DNA extraction method applied for the oat samples was successfully used for cereal samples including barley in a previous study by Sarlin et al. [16]. Samples were analyzed with the FusE qPCR assay, F. graminearium, and F. culmorumspecific qPCR assays, conventional Fusarium plating method, and DON measurement. The correlation of the FusE method with DON content was analyzed from the whole sample set. The Fusarium metabarcoding method was applied to a subset of 51 oat samples to which both high and low Fusarium-contaminated samples were selected from each year. Although we applied the FusE qPCR assay for cereal samples in this study, the method can be used to quantify Fusarium DNA in many different environmental samples (data not shown). The specificity of our assay was confirmed in silico, and subsequently against a diverse panel of fungal and bacterial species associated with cereals. Only Fusarium species, as well as Microdochium nivale and Microdochium majus, were amplified with the FusE qPCR assay. The cross-reaction between $M$. nivale strains was expected, since $M$. nivale is a close relative to Fusarium fungi and has previously been part of the Fusarium genus [21,22]. Cross-reaction was not detected with DNA extracted from sterile oat cells, revealing that the primers were not amplifying any regions of oat DNA. Furthermore, PCR-inhibitory effects were not 
detected when spiking a DNA sample isolated from oat grains with a known amount of Fusarium DNA.

The FusE qPCR method developed in this study proved as an effective tool to monitor Fusarium contamination in cereals. The FusE qPCR assay revealed that the amount of Fusarium DNA and thus the severity of Fusarium infection was notably higher in cereal samples collected in the years 2016 and 2017 compared to the samples collected in 2015. The amounts of Fusarium spp. DNA and F. graminearum DNA analyzed with the FusE and F. graminearum species-specific qPCR assays, respectively, correlated significantly with deoxynivalenol (DON) toxin content in each sampling year. F. culmorum DNA levels were very low in all years and no correlation was found between F. culmorum qPCR results and DON content. FusE qPCR method can be used to quantify the total Fusarium biomass to screen the mycotoxin risk in cereal samples. If Fusarium DNA is detected, then the presence and abundance of specific toxin-producing Fusarium species can be measured using species-specific Fusarium primers, such as F. graminearum or F. culmorum primers, to evaluate the mycotoxin risk more precisely $[8,23]$. In our study, the high correlation between the amounts of Fusarium DNA and DON content was detected, but in a study by Sarlin et al. [16], barley grain samples contained relatively high amounts of trichotheceneproducing Fusarium DNA, although no DON was detected. Some of the results presented by Sarlin et al. [16] were explained with the production of other trichothecenes than DON and low levels of $F$. graminearum, but also environmental factors such as temperature, humidity, and other microbes, and the host plant can influence the mycotoxin production of Fusarium species in cereals [24,25]. The effects of environmental factors can impact the estimation of mycotoxin risk based only on the Fusarium DNA levels. Another application for the FusE method is the assessment of the gushing risk in malting barley such as that studied by Virkajärvi et al. [26], where a higher Fusarium DNA level was associated with a higher gushing risk.

The economic losses caused by FHB to cereal producers are significant, and thus effective management strategies for FHB are needed. Important tools to monitor the FHB are Fusarium-specific detection and quantification methods. With these methods, the effectiveness of the management methods can be evaluated and mycotoxin risk in cereals can be assessed. In addition to the level of Fusarium contamination in cereals, the development of these management methods requires information about the composition of the whole Fusarium community because different populations could be affected selectively [13]. In this study, we evaluated the suitability of the new Fusarium metabarcoding method by Cobo-Díaz et al. [19] for Fusarium community profiling in cereal samples. With this new metabarcoding method, the whole Fusarium community in Finnish oat samples was characterized. Similar Fusarium species were detected with both the Fusarium plating method and metabarcoding methods. F. graminearum was detected especially in the oat samples collected in 2016 and 2017 according to both the plating method and metabarcoding methods. Only low amounts of F. culmorum were detected in the oat samples with plating and metabarcoding methods. F. oxysprorum was detected as the dominant Fusarium species in Finnish oat samples with metabarcoding. F. oxysporum was also the most abundant species in soil samples collected from maize fields in the study of Cobo et al. where the Fusarium metabarcoding method was established [19]. F. oxysporum was not detected in oat samples with the Fusarium plating method, and F. arthrosporioides and F. sambucinum were detected from oat samples by the plating method but not by the metabarcoding method. The plating method can favor some Fusarium species over others, and species growing faster can overtake other slower growing species, which can explain the differences in the results by the plating method and metabarcoding method. In addition, some sequences could not be identified to the species level with the metabarcoding method. This could be due to the lack of Fusarium elongator factor sequences in the sequence databases, or the fact that these species cannot be distinguished from each other because of sequence similarity in the elongation factor region between those taxa [19]. 
A shift from F. culmorum to F. graminearum in FHB infected cereal grains has been detected in Europe [10-12]. The results of this study confirm that F. graminearum was detected as the main mycotoxin-producing Fusarium species in Finnish oat samples. The reason for this shift is still partly unknown, but Parikka et al. [27] indicated that the shift is due to climate changes and farming practices. Climate is known to affect the Fusarium incidence and Fusarium community structure [28].

New Fusarium metabarcoding methods enable the detection of the Fusarium community to the species level and monitoring the distribution and community shifts in cereal production. In addition, new emerging species can be detected with these methods. Compared to the laborious and time-consuming plating method that needs high levels of expertise in Fusarium species identification, the new Fusarium metabarcoding method provides the Fusarium species information from cereal samples faster and more reliably. The Fusarium metabarcoding method by Cobo-Díaz et al. [19] detected Fusarium species as well as Microdochium nivale. M. nivale is a close relative to Fusarium species and has been previously part of the Fusarium genus, which explains the similarities in the targeted elongation factor region and amplification of $M$. nivale with primers Fa-150 and Ra2 designed by Cobo-Díaz et al. [19]. The Fusarium plating method is only a semi-quantitative method because the method only shows the percentage of the contaminated grains. With the Fusarium metabarcoding method, the relative abundance of the Fusarium species is obtained. Relative abundances that are currently used in metabarcoding studies can be compared within the same dataset, but this can lead to misinterpretations of microbial community structures. Because the sequencing data are compositional, the increase in one taxon leads to the concurrent decrease in the others [29]. Quantitative PCR provides the absolute quantification of the Fusarium DNA but does not give the species identification, and developing a species-specific qPCR method for all Fusarium species is not reasonable. Quantitative PCR combined with metabarcoding could be used in the absolute quantification of microbial abundances [29]. FusE qPCR assay combined with Fusarium metabarcoding method could be used in the absolute quantification of the whole Fusarium species complex in cereal samples.

Fusarium community structure in cereals can be studied in more detail using the novel Fusarium metabarcoding method. The significance of the whole cereal microbiome for the development of Fusarium-related diseases has been recognized lately. Both interactions within the Fusarium microbiome and interaction with the plant microbiome in the cereals play an important role in the FHB outbreaks and accumulation of mycotoxins [30]. Knowledge gained from these interactions can be used in developing new management methods for FHB. Future studies are especially needed to identify the characteristics of cereal microbiomes linked to Fusarium suppression.

\section{Materials and Methods}

\subsection{Materials}

The fungal and bacteria pure cultures used in this study were obtained from the culture collection of VTT Technical Research Centre of Finland Ltd. (VTT Culture Collection, Espoo, Finland) (Table 1). From the Fusarium genus, 14 different Fusarium species were used in the evaluation of the specificity of the developed FusE qPCR method. In addition, 12 other fungal strains commonly associated with cereals, one yeast strain, and two bacterial strains were tested. Fungal strains were cultured on potato dextrose agar (CM0139, Oxoid Ltd., Hampshire, UK), yeast strain on yeast mold agar (yeast: B271210, Becton, Dickinson and Company, Franklin Lakes, NJ, USA), and bacterial strains on MRS agar (de Man, Rogosa and Sharpe, CM0361, Oxoid Ltd., Hampshire, UK). Strains were incubated for 2-4 days at $25^{\circ} \mathrm{C}$ before the DNA was isolated.

\subsection{Field Samples}

Cereal samples were selected among the yearly crop survey samples collected around Finland by the Finnish Cereal Committee during the years 2015-2017. The total amount of 
the samples consisted of 157 oat samples and 40 barley samples. DON content detected in the cereal samples ranged from $<25$ to $26,000 \mu \mathrm{g} / \mathrm{kg}$.

In this paper, a subset of 51 oat samples was selected from the whole field sample set to compare the results of the new Fusarium-specific FusE qPCR method, the conventional Fusarium plating method, F. graminearum- and F. culmorum-specific qPCR methods and DON measurement. Both samples with high and low Fusarium contamination were selected in the subset. In addition, the new Fusarium-specific metabarcoding method was applied for the same subset of 51 oat samples. The correlation analysis was performed for the whole field sample set.

\subsection{DNA Isolation}

DNA from pure microbial isolates and ground cereal samples was extracted with FastDNA $^{\circledR}$ SPIN Kit for Soil (MP Biomedicals, Irvine, CA, USA). Cereal samples (10-20 g) were ground into fine flour with a grain mill (Bauknecht, Stuttgart, Germany). The DNA extraction was carried out from $100 \mathrm{mg}$ of microbial biomass of the pure cultures or grain flours according to the manufacturer's instructions but using a lysing step modified by VTT. The lysis was performed for $2 \times 1$ min at $6.0 \mathrm{~s}^{-1}$ using the FastPrep ${ }^{\circledR}$ Cell Disrupter (MP Biomedicals, Irvine, CA, USA) and Lysing Matrix A tubes (MP Biomedicals, Irvine, CA, USA). The tubes were placed on ice in between the two lysis runs. Centrifugation steps in the DNA extraction were carried out in Eppendorf Centrifuge 5424 (Eppendorf, Hamburg, Germany) and $13,000 \times g$. DNA was extracted in triplicate from each cereal sample. Extracted DNA was stored in a freezer at $-20^{\circ} \mathrm{C}$ until being further analyzed.

\subsection{Design of Primers and Hydrolysis Probe for Fusarium Genus}

Primers FusEF and FusER and probe FusEP for the FusE TaqMan qPCR were designed in silico (Table 2). The reference Fusarium elongation factor EF1 $\alpha$ gene sequences were searched from the JGI database (Joint Genome Institute, https://genome.jgi.doe. gov / portal/, accessed on 1 June 2017), Broad Institute (https:/ / www.broadinstitute.org/, accessed on 1 June 2017), and Ensemble Fungi (https:/ / fungi.ensembl.org/index.html, accessed on 1 June 2017). Specific regions to Fusarium species were identified using BLASTn search (NCBI, Bethesda, MD, USA, https:/ / blast.ncbi.nlm.nih.gov/Blast.cgi, accessed on 1 June 2017) against the NCBI nucleotide collection (nr/nt) (NCBI, Bethesda MD, USA, https:/ / www.ncbi.nlm.nih.gov/, accessed on 1 June 2017). Next, ClustalO (The European Bioinformatics Institute (EMBL-EBI), Cambridgeshire, United Kingdom https: / / www.ebi.ac.uk/Tools/msa/clustalo/, accessed on 1 June 2017) was used to align ten closest homologs. Several primer pairs binding to the Fusarium EF1 $\alpha$ gene were designed using Primer-BLAST (NCBI, Bethesda MD, USA, https: / / www.ncbi.nlm.nih.gov / tools / primer-blast/, accessed on 1 June 2017) and the NCBI nr database and a primer pair that amplified only Fusarium species were selected. The designed primers amplified a 96 bp region of the EF $1 \alpha$ gene, and the melting temperature of the primers was $59-68{ }^{\circ} \mathrm{C}$. The primers and the probe used in this study were synthesized by Integrated DNA Technologies (IDT Inc., Coralville, IA, USA). A reporter dye FAM (6-carboxy-fluorescein) was used in labeling the $5^{\prime}$ end and quencher dye TAMRA (6-carboxy-tetramethyl rhodamine) the $3^{\prime}$ end of the probe FusEP.

Table 2. Sequences of the primers (FusEF, FusER) and probe (FusEP) of the FusE TaqMan qPCR to amplify the Fusarium spp.

\begin{tabular}{cc}
\hline Primer/Probe & Sequence $\left(\mathbf{5}^{\prime}\right.$ to $\mathbf{3}^{\prime}$ ) \\
\hline FusEF (forward) & CTGGGTTCTTGACAAGCTCA \\
FusER (reverse) & CGGTGACATAGTAGCGAGGA \\
FusEP (probe) & TACCACGCTCACGCTCGGCT \\
\hline
\end{tabular}




\subsection{TaqMan qPCR and Data Analysis}

qPCR amplification was performed in the LightCycler ${ }^{\circledR} 480$ qPCR device (Roche Diagnostics Ltd., Risch-Rotkreuz, Switzerland) with software version Version 1.5.0.39. PCR amplification was carried out in $20 \mu \mathrm{L}$ volume reactions using the Lightcycler 480 Probes Master Kit (Roche Molecular Systems Inc., Pleasanton, CA, USA), containing $2 \times$ Probes Master, 5 pmol of each primer, 2 pmol of the hydrolysis probe, and $5 \mu \mathrm{L}$ of the template. For microbial pure cultures concentration of $2 \mathrm{ng} \mu \mathrm{L}^{-1}$ was used. The PCR program for the FusE qPCR consisted of an initial denaturation step for $10 \mathrm{~min}$ at $95^{\circ} \mathrm{C}$, followed by 39 cycles of denaturation for $10 \mathrm{~s}$ at $95^{\circ} \mathrm{C}$, annealing for $45 \mathrm{~s}$ at $57^{\circ} \mathrm{C}$, and extension for $1 \mathrm{~s}$ at $72{ }^{\circ} \mathrm{C}$. In addition, negative controls with only PCR-grade water as a template were performed to rule out possible contamination. The F. graminearum-specific qPCR runs were carried out according to Yli-Mattila et al. [23], and F. culmorum-specific qPCR runs were carried out according to Waalwijk et al. [8].

The size of the amplified product was confirmed by loading the PCR products on $1.5 \%$ $(w / v)$ agarose gel stained with Midori Green Nucleic Acid Stain (Nippon Genetics Europe, Düren, Germany) and using a DNA ladder (GeneRuler, Thermo Fisher Scientific, Waltham, Massachusetts, USA).

A standard curve was set up using a serial dilution of 0.001 to $1 \mathrm{ng}$ target DNA per reaction of Fusarium DNA extracted from a pure culture of F. culmorum (VTT D-80148). The concentration of the F. culmorum DNA was quantified spectrophotometrically using a NanoDrop 2000 system (Thermo Scientific, Waltham, Massachusetts, USA). Three replicates were measured for each standard dilution.

The PCR inhibition of the cereal matrix was studied by spiking DNA extracted from a sterile oat sample with $1 \mathrm{ng}$ of Fusarium genomic DNA. PCR reactions were performed in triplicate, as described above. The cycle threshold $(\mathrm{Ct})$ values between the spiked reactions and Fusarium standard were compared to investigate potential PCR inhibition. The results obtained from the real-time PCR assay were analyzed with Abs Quant/2nd Derivate Max method in LightCycler ${ }^{\circledR} 480$ program (Roche Diagnostics Ltd., Risch-Rotkreuz, Switzerland), and the results were normalized by dividing the Fusarium DNA quantities with the amounts of total genomic DNA in the template.

\subsection{Conventional Fusarium Plating from Field Samples}

Fusarium contamination in cereal samples was determined by plating (100 grains/sample) on peptone-pentachloronitrobenzene (PCNB) medium (Nash and Snyder medium) [31] and incubating the plates in the dark at room temperature $\left(22^{\circ} \mathrm{C}\right)$. The growing colonies were isolated on potato dextrose agar (PDA), and Fusarium species were identified morphologically from the developing colonies. The percentage of grains contaminated with each identified Fusarium species was calculated.

\subsection{DON Analysis}

Trichothecenes were analyzed as described by Hietaniemi et al. [9]. The laboratories of Natural Resources Institute Finland apply a quality control system in accordance with SFS-EN ISO/IEC 17025:2017. In brief, $15 \mathrm{~g}$ ground cereal samples were extracted with 84\% acetonitrile. The raw extract was purified with MycoSep \#227 SPE column (RomerLabs, Getzersdorf, Austria). The cleaned-up extract was transferred to a silylated test tube and evaporated to dryness. Deoxynivalenol (DON) was identified and quantified as trimethylsilylether derivatives by GC-MS. The limit of quantification (LOQ) was $25 \mu \mathrm{g} / \mathrm{kg}$. The method for trichothecenes (DON) has been accredited since 2003. The reference materials for DON quality control were either corn naturally contaminated with deoxynivalenol (Trilogy, TR-D100, $1.9 \pm 0.1 \mathrm{mg} / \mathrm{kg}$, Trilogy Washington, MO, USA) or matrix reference material deoxynivalenol in wheat (Biopure, BRM003022, $877 \mu \mathrm{g} / \mathrm{kg} \pm 23 \mu \mathrm{g} / \mathrm{kg}$ and QCM2W1, $906 \pm 68 \mu \mathrm{g} / \mathrm{kg}$, Biopure, Cambridge, MA, USA). 


\subsection{Fusarium Community Analysis with NGS}

DNA elutions extracted from 51 oat samples were sent to Illumina Miseq sequencing of the Fusarium elongation factor $(E F 1 \alpha)$ region to Microsynth AG, Switzerland. First, Fusarium EF1 $\alpha$ libraries were sequenced with Nextera (Illumina, Inc., San Diego, CA, USA) two-step PCR with primers Fa-150 5' CCGGTCACTTGATCTACCAG-3' and Ra-2 $5^{\prime}$ ATGACGGTGACATAGTAGCG-3' [19]. The libraries were sequenced at Microsynth AG by $2 \times 250$ bp paired-end sequencing on the MiSeq platform using MiSeq v3 Reagent Kit. Negative controls for amplicon barcoding PCR and positive controls including the known amount of DNA from species belonging to the genus Fusarium and a few other fungal species isolated from the cereal matrix were included in the sequencing set.

The sequence reads obtained from Illumina Miseq sequencing were subjected to sequence analysis using the DADA2 software package version 1.14 [32] and DADA2 Pipeline Tutorial 1.16 with some modifications. DADA2 package run in RStudio (RStudio, Boston, MA, USA, version 1.4.1106) with R version 4.0.4. First, the sequences were prefiltered to remove ambiguous bases (Ns) that could affect accurate mapping. Then, primers were identified from the sequences and removed using the cutadapt tool [33]. The quality of the sequence reads was checked according to the DADA2 workflow. Next sequences were filtered and trimmed using DADA2 filterAndTrim function. Filtering parameters $\max N=0, \operatorname{maxEE}=c(2,2)$, truncQ $=2, \operatorname{minLen}=50$ were used. A minimum length of $50 \mathrm{bp}$ was used to remove spurious very low-length sequences. The maximum possible error rates were calculated using the learnErrors command. Identical reads were de-replicated (unique sequences). Amplicon sequence variants of the sequence data were identified using the DADA2 pipelines core sample inference algorithm. Denoised paired reads were merged according to the DADA2 pipeline, and an amplicon sequence variant table (ASV) table was constructed. Subsequently, chimeric sequence reads were removed from the dataset with the removeBimeraDeNovo function, using the consensus option. Finally, taxonomy from the domain to the species level was assigned to ASVs with DADA2's native implementation of the naive Bayesian classifier method. Taxonomy was assigned against an in-house-generated Fusarium database for EF1 $\alpha$ sequences. The Fusarium database for EF1 $\alpha$ sequences was constructed according to Boutigny et al. [18], and in addition, sequences from Cobo-Díaz et al. [19] were added to the database. All images of the sequencing data were constructed with $\mathrm{R}$ using the packages phyloseq [34] and ggplot2 [35]. The Fusarium $E F 1 \alpha$ gene region sequences have been submitted to the European Nucleotide Archive (ENA, https: / / www.ebi.ac.uk/ena/, accessed on 31 July 2021) under accession numbers ERS7652901-ERS7652951.

\subsection{Statistical Analysis}

The correlation analysis to evaluate the correlation between qPCR results, DON content, and conventional Fusarium plating method was calculated using the IBM SPSS Statistics for Windows program (IBM Corp., Armonk, NY, USA, version 24). The Pearson or Spearman correlation coefficients at significance levels of $p<0.05$ and $p<0.01$ were calculated. Standard deviations of the qPCR results were calculated with Microsoft Excel (Microsoft Corp., Redmond, WA, USA, version 2102). Linear regression between Ct values and the amount of Fusarium DNA (ng) was calculated to acquire the $\mathrm{r}^{2}$ values of the qPCR standard curve. The qPCR, Fusarium plating method, and metabarcoding figures were created with $\mathrm{R}$ and correlation figures with Excel.

Supplementary Materials: The following are available online at https:/ /www.mdpi.com/article/10 .3390 / toxins14010045/s1, Figure S1: Relative abundance of all the species, including Microdochium nivale, detected with the Fusarium metabarcoding method.

Author Contributions: Conceptualization A.L., T.S.; methodology, V.V., E.S., A.L., T.S. and S.R.; validation, V.V. and E.S.; data curation, E.S., V.V. and T.S.; formal analysis, E.S. and V.V.; writingoriginal draft preparation, E.S., T.S. and S.R.; writing—review and editing, E.S., T.S., A.L., P.P. and S.R.; visualization, E.S., supervision, T.S. and A.L.; project administration, T.S. and A.L.; funding 
acquisition, T.S., A.L. and P.P. All authors have read and agreed to the published version of the manuscript.

Funding: This research was funded by the Development Fund for Agriculture and Forestry (Makera), (Doncontrol:1835/312/2014;1692/03.0.1.0.2/2015; Bioteho:536/03.01.02/2017), Technical Research Centre of Finland Ltd., Natural Resources Institute Finland, Boreal Plant Breeding Ltd. and PBL Brewing Laboratory.

Institutional Review Board Statement: Not applicable.

Informed Consent Statement: Not applicable.

Data Availability Statement: The NGS data that support the findings of this study are openly available in European Nucleotide Archive (ENA) at https: / / www.ebi.ac.uk/ under accession numbers ERS7652901-ERS7652951.

Acknowledgments: Leena Holkeri, Riitta Henriksson, and Kirsi Puisto from Luke are thanked for skillful trichothecene analysis. Ulla Holopainen-Mantila from VTT Ltd is thanked for SPSS analysis and Jenni Limnell for Fusarium qPCR analysis. Alishum Ali from Curtin University is thanked for the advice in making the Fusarium EF1 $\alpha$ sequence database for NGS analysis.

Conflicts of Interest: The authors declare no conflict of interest.

\section{References}

1. Golinski, P.; Kaczmarek, Z.; Kiecana, I.; Wisniewska, H.; Kaptur, P.; Kostecki, M.; Chelkowski, J. Fusarium Head Blight of Common Polish Winter Wheat Cultivars-Comparison of Effects of Fusarium avenaceum and Fusarium culmorum on Yield Components. J. Phytopathol. 2002, 150, 135-141. [CrossRef]

2. Nganje, W.E.; Kaitibie, S.; Wilson, W.W.; Leistritz, F.L.; Bangsund, D.A. Economic Impacts of Fusarium Head Blight in Wheat and Barley: 1993-2001; Agribusiness and Applied Economics: Fargo, ND, USA, 2004.

3. Wilson, W.; Dahl, B.; Nganje, W. Economic costs of Fusarium Head Blight, scab and deoxynivalenol. World Mycotoxin J. 2018, 11, 291-302. [CrossRef]

4. Parry, D.W.; Jenkinson, P.; McLeod, L. Fusarium ear blight (scab) in small grain cereals-A review. Plant Pathol. 1995, 44, 207-238. [CrossRef]

5. Morcia, C.; Tumino, G.; Gasparo, G.; Ceresoli, C.; Fattorini, C.; Ghizzoni, R.; Carnevali, P.; Terzi, V. Moving from qPCR to Chip Digital PCR Assays for Tracking of some Fusarium Species Causing Fusarium Head Blight in Cereals. Microorganisms 2020, 8 , 1307. [CrossRef]

6. Aoki, T.; O'Donnell, K.; Geiser, D.M. Systematics of key phytopathogenic Fusarium species: Current status and future challenges. J. Gen. Plant Pathol. 2014, 80, 189-201. [CrossRef]

7. Ji, F.; He, D.; Olaniran, A.O.; Mokoena, M.P.; Xu, J.; Shi, J. Occurrence, toxicity, production and detection of Fusarium mycotoxin: A review. Food Prod. Process. Nutr. 2019, 1, 1-14. [CrossRef]

8. Waalwijk, C.; Van Der Heide, R.; De Vries, I.; Van Der Lee, T.; Schoen, C.; Costrel-De Corainville, G.; Haüser-Hahn, I.; Kastelein, P.; Rgen, J.; Köhl, K.; et al. Quantitative detection of Fusarium species in wheat using TaqMan. Eur. J. Plant Pathol. 2004, 110, 481-494. [CrossRef]

9. Hietaniemi, V.; Rämö, S.; Yli-Mattila, T.; Jestoi, M.; Peltonen, S.; Kartio, M.; Sieviläinen, E.; Koivisto, T.; Parikka, P. Updated survey of Fusarium species and toxins in Finnish cereal grains. Food Addit. Contam. Part A 2016, 33, 831-848. [CrossRef]

10. Klix, M.B.; Beyer, M.; Verreet, J.-A. Effects of cultivar, agronomic practices, geographic location, and meteorological conditions on the composition of selected Fusarium species on wheat heads. Can. J. Plant Pathol. 2010, 30, 46-57. [CrossRef]

11. Nielsen, L.K.; Jensen, J.D.; Nielsen, G.C.; Jensen, J.E.; Spliid, N.H.; Thomsen, I.K.; Justesen, A.F.; Collinge, D.B.; Jørgensen, L.N. Fusarium Head Blight of Cereals in Denmark: Species Complex and Related Mycotoxins. Phytopathology 2011, 101, 960-969. [CrossRef] [PubMed]

12. Karlsson, I.; Friberg, H.; Kolseth, A.K.; Steinberg, C.; Persson, P. Agricultural factors affecting Fusarium communities in wheat kernels. Int. J. Food Microbiol. 2017, 252, 53-60. [CrossRef] [PubMed]

13. Valverde-Bogantes, E.; Bianchini, A.; Herr, J.R.; Rose, D.J.; Wegulo, S.N.; Hallen-Adams, H.E. Recent population changes of Fusarium head blight pathogens: Drivers and implications. Can. J. Plant Pathol. 2019, 42, 315-329. [CrossRef]

14. Karelov, A.V.; Borzykh, O.I.; Kozub, N.O.; Sozinov, I.O.; Yanse, L.A.; Sozinova, O.I.; Tkalenko, H.M.; Mishchenko, L.T.; Blume, Y.B. Current Approaches to Identification of Fusarium Fungi Infecting Wheat. Cytol. Genet. 2021, 55, 433-446. [CrossRef]

15. Bluhm, B.H.; Cousin, M.A.; Woloshuk, C.P. Multiplex Real-Time PCR Detection of Fumonisin-Producing and TrichotheceneProducing Groups of Fusarium Species. J. Food Prot. 2004, 67, 536-543. [CrossRef]

16. Sarlin, T.; Yli-Mattila, T.; Jestoi, M.; Rizzo, A.; Paavanen-Huhtala, S.; Haikara, A. Real-time PCR for quantification of toxigenic Fusarium species in barley and malt. Eur. J. Plant Pathol. 2006, 114, 371-380. [CrossRef] 
17. Kulik, T.; Jestoi, M.; Okorski, A. Development of TaqMan assays for the quantitative detection of Fusarium avenaceum/Fusarium tricinctum and Fusarium poae esyn1 genotypes from cereal grain. FEMS Microbiol. Lett. 2011, 314, 49-56. [CrossRef] [PubMed]

18. Boutigny, A.L.; Gautier, A.; Basler, R.; Dauthieux, F.; Leite, S.; Valade, R.; Aguayo, J.; Ioos, R.; Laval, V. Metabarcoding targeting the EF1 alpha region to assess fusarium diversity on cereals. PLoS ONE 2019, 14, e0207988. [CrossRef] [PubMed]

19. Cobo-Díaz, J.F.; Baroncelli, R.; Le Floch, G.; Picot, A. A novel metabarcoding approach to investigate Fusarium species composition in soil and plant samples. FEMS Microbiol. Ecol. 2019, 95, fiz084. [CrossRef]

20. Stępniewska, H.; Jankowiak, R.; Bilański, P.; Hausner, G. Structure and abundance of fusarium communities inhabiting the litter of beech forests in central europe. Forests 2021, 12, 811. [CrossRef]

21. Wollenweber, H.W. Fusarium monograph. Parasitic and saprophytic fungi. Z. Parasitenkd. 1931, 3, 269-516. [CrossRef]

22. Glynn, N.C.; Hare, M.C.; Parry, D.W.; Edwards, S.G. Phylogenetic analysis of EF-1 alpha gene sequences from isolates of Microdochium nivale leads to elevation of varieties majus and nivale to species status. Mycol. Res. 2005, 109, 872-880. [CrossRef] [PubMed]

23. Yli-Mattila, T.; Paavanen-Huhtala, S.; Jestoi, M.; Parikka, P.; Hietaniemi, V.; Gagkaeva, T.; Sarlin, T.; Haikara, A.; Laaksonen, S.; Rizzo, A. Real-time PCR detection and quantification of Fusarium poae, F. graminearum, F. sporotrichioides and F. langsethiae in cereal grains in Finland and Russia. Arch. Phytopathol. Plant Prot. 2008, 41, 243-260. [CrossRef]

24. Doohan, F.M.; Brennan, J.; Cooke, B.M. Influence of climatic factors on Fusarium species pathogenic to cereals. In Epidemiology of Mycotoxin Producing Fungi; Springer: Dordrecht, The Netherlands, 2003; pp. 755-768. [CrossRef]

25. Brennan, J.M.; Egan, D.; Cooke, B.M.; Doohan, F.M. Effect of temperature on the head blight of wheat caused by Fusarium culmorum and F. graminearum. Plant Pathol. 2005, 54, 156-160. [CrossRef]

26. Virkajärvi, V.; Sarlin, T.; Laitila, A. Fusarium profiling and barley malt gushing propensity. J. Am. Soc. Brew. Chem. 2017, 75, 181-192. [CrossRef]

27. Parikka, P.; Hakala, K.; Tiilikkala, K. Expected shifts in Fusarium species' composition on cereal grain in Northern Europe due to climatic change. Food Addit. Contam. Part A Chem. Anal. Control. Expo. Risk Assess. 2012, 29, 1543-1555. [CrossRef]

28. Del Ponte, E.M.; Fernandes, J.M.C.; Pavan, W.; Baethgen, W.E. A Model-based Assessment of the Impacts of Climate Variability on Fusarium Head Blight Seasonal Risk in Southern Brazil. J. Phytopathol. 2009, 157, 675-681. [CrossRef]

29. Jian, C.; Luukkonen, P.; Yki-Järvinen, H.; Salonen, A.; Korpela, K. Quantitative PCR provides a simple and accessible method for quantitative microbiota profiling. PLoS ONE 2020, 15, e0227285. [CrossRef]

30. Karlsson, I.; Persson, P.; Friberg, H. Fusarium Head Blight from a Microbiome Perspective. Front. Microbiol. $2021,12,371$ [CrossRef]

31. Nelson, P.E.; Toussoun, T.A. Marasas WFO Fusarium Species: An Illustrated Manual for Identification; University Park, Pennsylvania State University Press: Philadelphia, PA, USA, 1983.

32. Callahan, B.J.; McMurdie, P.J.; Rosen, M.J.; Han, A.W.; Johnson, A.J.A.; Holmes, S.P. DADA2: High-resolution sample inference from Illumina amplicon data. Nat. Methods 2016, 13, 581-583. [CrossRef]

33. Martin, M. Cutadapt removes adapter sequences from high-throughput sequencing reads. EMBnet J. 2011, 17, 10. [CrossRef]

34. McMurdie, P.J.; Holmes, S. Phyloseq: An R Package for Reproducible Interactive Analysis and Graphics of Microbiome Census Data. PLoS ONE 2013, 8, e61217. [CrossRef]

35. Wicham, H. ggplot2: Elegant Graphics for Data Analysis 2016; Springer: New York, NY, USA, 2016; ISBN 978-3-319-24277-4. 